\title{
WiP Abstract: Pseudorange Measurement Outlier Detection for Navigation with Cellular Signals
}

\author{
Mahdi Maaref \\ University of California, Irvine \\ Irvine, California \\ mmaaref@uci.edu
}

\author{
Joe Khalife \\ University of California, Irvine \\ Irvine, California \\ khalifej@uci.edu
}

\author{
Zaher M. Kassas \\ University of California, Irvine \\ Irvine, California \\ zkassas@ieee.org
}

\begin{abstract}
We present an autonomous measurement outlier detection and exclusion framework for ground vehicle navigation using cellular signals of opportunity (SOPs) and an inertial measurement unit (IMU). The experimental results demonstrate the proposed framework successfully detecting and excluding outlier measurements, improving the position root mean-squared error (RMSE) by $42 \%$. The demo session will showcase work in progress, namely (1) demo (in the form of a video of our experiment driving in downtown Riverside, California) and (2) a poster that includes the navigation framework, the proposed outlier detection method, and the experimental results.
\end{abstract}

\section{ACM Reference Format:}

Mahdi Maaref, Joe Khalife, and Zaher M. Kassas. 2019. WiP Abstract: Pseudorange Measurement Outlier Detection for Navigation with Cellular Signals . In Proceedings of ICCPS '19,. ACM, Montreal, QC, Canada, Article 24, 2 pages. https://doi.org/https://doi.org/10.1145/3302509.3313334

\section{INTRODUCTION}

Global navigation satellite system (GNSS) signals are insufficient for reliable and accurate ground vehicle navigation in deep urban environments due to the inherent weakness of their spacebased signals. Among alternative sensing modalities to compensate for GNSS limitations and vulnerabilities, signals of opportunity (SOPs) represent a particularly fruitful class of sensing modalities $\mathrm{AT}_{\mathrm{E}} \mathrm{X}$ [2]. SOPs are radio frequency signals that are not intended for navigation but can be exploited for navigation purposes, such as AM/FM radio signals, digital television, cellular, and low Earth orbit (LEO) satellites.

Despite the promise of SOPs as a reliable and accurate sensing modality, their integrity has not been fully studied. An initial work on integrity monitoring for SOP-based navigation was conducted in $\mathrm{LT}_{\mathrm{E}} \mathrm{X}$ [3]. This paper extends the work in $\mathrm{ET}_{\mathrm{E}} \mathrm{X}$ [3] by developing an autonomous measurement outlier detection and exclusion method for cellular long-term evolution (LTE) SOPs due to two main sources of errors: line-of-sight (LOS) blockage and short multipath delays. Experimental results are presented validating the efficacy of the proposed method for a ground vehicle navigating in an urban environment (downtown Riverside, California) over a trajectory of $1.4 \mathrm{~km}$. The position root mean-squared error (RMSE) is

Permission to make digital or hard copies of part or all of this work for personal or classroom use is granted without fee provided that copies are not made or distributed for profit or commercial advantage and that copies bear this notice and the full citation on the first page. Copyrights for third-party components of this work must be honored.

For all other uses, contact the owner/author(s).

ICCPS '19,, April 16-18, Montreal, QC, Canada

(C) 2019 Copyright held by the owner/author(s).

ACM ISBN 978-1-4503-6285-6/19/04.

https://doi.org/https://doi.org/10.1145/3302509.3313334 reduced by $42 \%$ when the measurement outliers are detected and excluded via the proposed method.

\section{NAVIGATION FRAMEWORK}

The environment is assumed to comprise $N_{s}$ terrestrial cellular transmitters, denoted $\left\{S_{n}\right\}_{n=1}^{N_{s}}$ and the vehicle is assumed to be equipped with an inertial measurement unit (IMU) and a receiver capable of producing pseudorange measurements to cellular transmitters. It is assumed that the vehicle knows the location of the cellular transmitters (e.g., from a local or a cloud-hosted database). It is also assumed that the vehicle has an initial period of access to GNSS signals. During this period, the vehicle estimates its state. After this period, it is assumed that GNSS signals become unusable, and the vehicle begins to navigate exclusively with cellular signals and the IMU. In addition, the proposed framework assumes the presence of an stationary agent in the vehicle's environment, referred to as the base, which has knowledge of its own state at all time. The base's purpose is to estimate the dynamic stochastic clock bias states of cellular transmitters and to share these estimates with the navigating vehicle. The cellular receiver draws pseudorange observations from each cellular transmitters, given by

$$
z_{s_{n}}(k)=\left\|\boldsymbol{r}_{r}(k)-\boldsymbol{r}_{s_{n}}\right\|_{2}+c\left[\delta t_{r}(k)-\delta t_{s_{n}}(k)\right]+v_{s_{n}}(k),
$$

where $\boldsymbol{r}_{r}$ and $\boldsymbol{r}_{s_{n}}$ are the location of the receiver and $n$-th cellular transmitter, respectively; $\delta t_{r}$ and $\delta t_{s_{n}}$ represent the clock bias of the receiver and $n$-th cellular transmitter; and $v_{s_{n}}$ is the measurement noise, which is modeled as a discrete-time zero-mean white Gaussian sequence. The clock biases of the cellular transmitters $\left\{\delta t_{s_{n}}\right\}_{n=1}^{N_{s}}$ are known to the navigating vehicle through a base receiver. The observations $\left\{z_{s_{n}}\right\}_{n=1}^{N_{s}}$ are fused through an extended Kalman filter (EKF), which produces an estimate of the receiver's state vector $\hat{x}$ and an associated estimation error covariance $P$. The EKF-based estimation framework is illustrated in Figure 1.

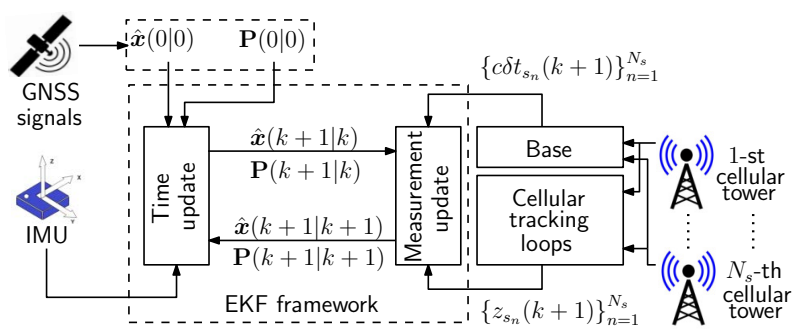

Figure 1: EKF-based estimation framework to fuse IMU data with cellular. 


\section{OUTLIER DETECTION}

In the presence of a measurement bias due to LOS signal blockage or due to short multipath delays, a bias with magnitude $b_{n}$ is injected in the pseudorange measurement drawn from the $n$-th cellular transmitter, rendering that particular measurement an outlier. In order to distinguish between outlier-free measurements and those subject to outliers, a measurable scalar parameter is defined that provides information about pseudorange measurement errors. This parameter, called a test statistic, is a random variable with a known distribution. Under outlier-free, normal operation, the innovation vector $\boldsymbol{v}_{0}(k+1)$ and its associated innovation error covariance $\mathrm{S}(k+1)$ are given by

$$
\begin{aligned}
\boldsymbol{v}_{0}(k+1) \triangleq z(k+1)-\hat{z}(k+1 \mid k) \approx \mathbf{H}(k+1) \hat{\boldsymbol{x}}(k+1 \mid k)+\boldsymbol{v}_{s}(k+1), \\
\mathbf{S}(k+1) \triangleq \mathbf{H}(k+1) \mathbf{P}(k+1 \mid k) \mathbf{H}^{\mathrm{T}}(k+1)+\mathbf{R},
\end{aligned}
$$

where $\boldsymbol{v}_{s} \triangleq\left[v_{s_{1}}, \ldots, v_{s_{N_{s}}}\right]^{\mathrm{T}}, \mathbf{H}$ is the measurement Jacobian, $\boldsymbol{z}$ is the vector of pseudorange measurements, and $\mathrm{R}$ is the measurement noise covariance matrix. Whenever the $n$-th measurement experiences an outlier bias, the biased innovation vector $\bar{v}(k+1)$ may be expressed as

$$
\overline{\boldsymbol{v}}(k+1)=\boldsymbol{v}_{0}(k+1)+\boldsymbol{u}_{n}(k+1),
$$

where the vector $\boldsymbol{u}_{n}(k+1) \triangleq\left[0, \ldots, 0, b_{n}(k+1), 0, \ldots, 0\right]^{\top}$ is the bias vector resulting when a bias of magnitude $b_{n}$ is present in the pseudorange measurement drawn from the outlier cellular tower. Note that $\bar{v}(k+1)$ has the same covariance as $\boldsymbol{v}_{0}(k+1)$. Denote $\boldsymbol{v}(k+$ 1) the innovation vector evaluated by the filter. The hypothesis test relies on the normalized innovation squared (NIS)-based test statistic, which is defined as

$$
\varphi(k+1) \triangleq v^{\top}(k+1) \mathrm{S}^{-1}(k+1) v(k+1) .
$$

Note that the test statistics follows a chi-square distribution under outlier-free operation and a non-central chi-square distribution in the presence of outliers $\mathrm{ATEX}_{\mathrm{E}}$ [1]. The degrees of freedom of the distributions under both conditions is $d=N_{s}$. Outlier detection is achieved by comparing $\varphi(k+1)$ against a detection threshold $T_{h}$, namely

$$
\begin{aligned}
& \varphi(k+1) \leq T_{h}: \text { no outliers present } \\
& \varphi(k+1)>T_{h}: \text { outlier present. }
\end{aligned}
$$

\section{EXPERIMENTAL RESULTS}

A vehicle was equipped with an IMU and a cellular receiver (Figure 2). Over the course of the experiment, the vehicle-mounted receiver was listening to 5 LTE base stations. The outlier detection test was performed throughout the experiment. The results are shown in Figure 3 and Figure 4. Figure 3 shows the outlier detection test which compares the test statistic $\varphi$ against the detection threshold $T_{h}$. It can be seen that at $t=95 \mathrm{~s}$, the threshold is exceeded; therefore, the test is not declared successful (see the red circle in Figure 3). This implies that at least one of the measurements was detected as an outlier and its contribution to the test statistic was significant enough for the test to fail. Figure 4 shows the resulting position estimation error and corresponding $\pm 3 \sigma$ bounds with and without using the proposed outlier exclusion. As can be seen, outlier exclusion results in a significant reduction in the $x$ -

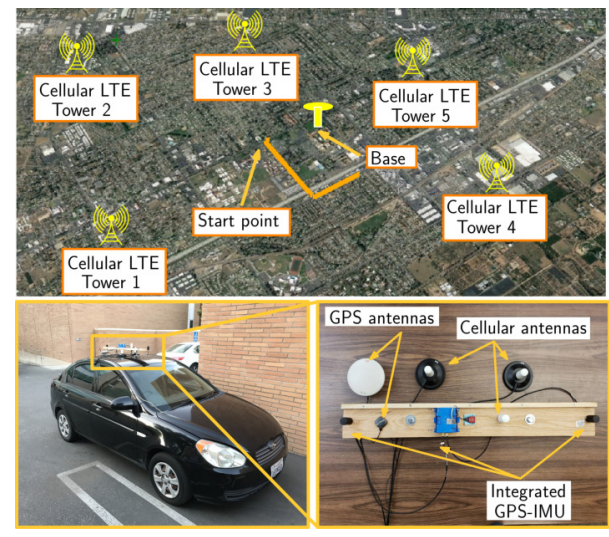

Figure 2: Experimental hardware setup and the traversed trajectory along with the position of cellular towers.

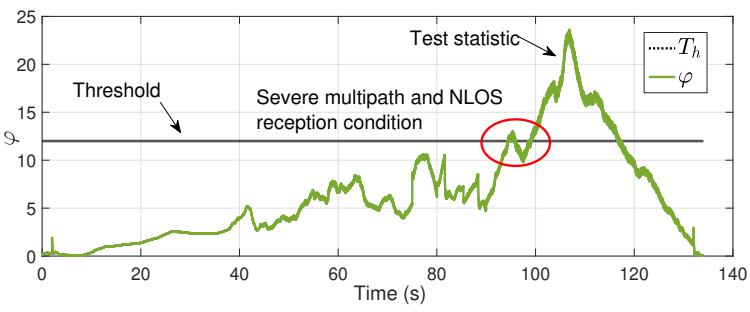

Figure 3: The resulting fault detection test.

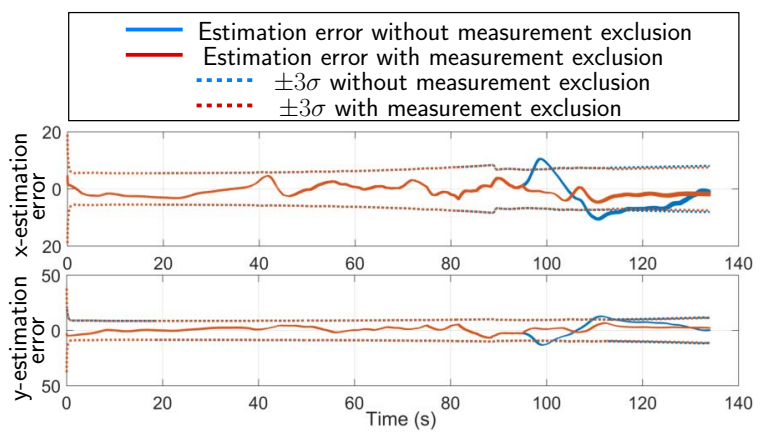

Figure 4: Vehicle's position estimation errors in the $x$ - and $y$ directions with and without the proposed autonomous measurement outlier exclusion method.

and $y$-direction estimation error. The position RMSE without measurement exclusion was $8.2 \mathrm{~m}$, whereas the position RMSE with the proposed autonomous measurement outlier exclusion method was $4.8 \mathrm{~m}$. Hence, incorporating the proposed algorithm reduced the position RMSE by $42 \%$.

\section{REFERENCES}

[1] S. Bhattacharyya and D. Gebre-Egziabher. 2015. Kalman filter-based RAIM for GNSS receivers. IEEE Trans. Aerospace Electron. Systems 51, 3 (July 2015), 24442459.

[2] Z. Kassas. 2014. Analysis and Synthesis of Collaborative Opportunistic Navigation Systems. Ph.D. Dissertation. The University of Texas at Austin, USA.

[3] M. Maaref, J. Khalife, and Z. Kassas. 2018. Integrity monitoring of LTE signal of opportunity-based navigation for autonomous ground vehicles. In Proceedings of ION GNSS Conference. 2456-2466. 